\section{Tuberculose: subnotificação de casos que evoluíram para o óbito em Fortaleza-CE}

\section{Tuberculosis: underreporting of tuberculosis cases that resulted in death in Fortaleza-CE}

\section{Mônica Cardoso Façanha}

Departamento de Saúde Comunitária, Faculdade de Medicina, Universidade Federal do Ceará

\section{Resumo}

O Plano Nacional de Controle da Tuberculose tem como meta diagnosticar pelo menos $92 \%$ dos casos esperados, e tratar com sucesso no mínimo $85 \%$ deles. O acompanhamento dessas metas depende da informação dos casos diagnosticados e de sua evolução. O objetivo deste estudo é verificar se existe subnotificação de casos de tuberculose que evoluíram para o óbito. Este é um estudo descritivo de dados secundários em que foi feita busca no Sistema de Informação de Agravos de Notificação (Sinan) dos óbitos ocorridos entre 1999 e 2003, que tiveram tuberculose como uma das causas múltiplas e estavam registrados no Sistema de Informação em Mortalidade (SIM), tendo como variáveis de referência o nome, data do nascimento e nome da mãe. Dos óbitos registrados, 610 tiveram tuberculose como uma das causas associadas, $204(33,4 \%)$ deles notificados no Sinan. Em 438 (71,8\%), a tuberculose foi causa básica. A média de notificações entre 1999 e 2002 foi de 37\%, reduzindo-se para 19,3\% em 2003 ( $\mathrm{p}=0,03)$. Em cinco hospitais ocorreram 324 (53,1\%) dos óbitos e tiveram origem 114 (55,9\%) notificações. O Sinan registrou 153 evoluções para o óbito. O acréscimo da notificação dos 406 casos de tuberculose que evoluíram para o óbito acarretará incremento de 5,9\% no total de casos notificados no período e de $265,4 \%$ nas evoluções para o óbito. Os casos graves de tuberculose encontram-se subnotificados, sendo o SIM uma importante fonte de resgate desses casos, que pode ser utilizada de forma rotineira e preferencialmente informatizada.

Palavras-chave: Tuberculose. Vigilância epidemiológica. Sistemas de informação. Mortalidade. 


\section{Abstract}

The National Tuberculosis Control Plan aims to diagnosis at least $92 \%$ of expected cases and the successful treatment of $85 \%$ of cases of tuberculosis. Information on diagnosed cases and outcome is essential to know if the Plan has been followed. This study aims to verify if patients that died of tuberculosis were underreported. This is a descriptive study that searched the National Surveillance Diseases System - Sinan for secondary data on tuberculosis cases that led to death between 1999 and 2003 and that were registered in the Mortality Information System SIM of Fortaleza (Northeastern Brazilian city). Variables for comparison were names, dates of birth and mothers' names. Of all registered deaths, 610 had tuberculosis as one of the causes and 204 (33.4\%) were found in Sinan. Rescuing these 406 cases result in a $5.9 \%$ increase in total tuberculosis cases reported in Sinan. Sinan registered 153 tuberculosis cases that resulted in death, and an additional reporting of 406 cases increases cases that resulted in death by $265.4 \%$. Tuberculosis was the basic cause in 438 (71.8\%). Reported cases varied from $39 \%$ in 2001 to $19 \%$ in $2003(\mathrm{p}=0.03)$. Three hundred and twenty-four (53.1\%) deaths occurred in five hospitals that led to 114 (55.9\%) cases reported. Tuberculosis cases that resulted in death were underreported and SIM was an important source of rescuing severe cases and should be used more frequently.

Key Words: Tuberculosis. Epidemiologic surveillance. Health Systems information. Mortality.

\section{Introdução}

Em 1993, a Organização Mundial da Saúde declarou a situação da tuberculose como uma emergência mundial devido ao recrudescimento da doença nos países desenvolvidos, onde se previa que estivesse sob controle, e à expansão nos continentes asiático e americano. A OMS atribuiu a gravidade da situação à desigualdade social, ao advento da Aids, ao envelhecimento da população e aos grandes movimentos migratórios. No Brasil, a tuberculose manteve-se endêmica, mesmo quando nos países industrializados era considerada sob controle ${ }^{1}$. No Brasil e no Ceará, o coeficiente de mortalidade variou de 3,6 em 1999 para 3,0 em 2002, e em Fortaleza de 4,5 para 4,4 óbitos por cem mil habitantes ${ }^{2}$.

Desde o século XIX, o Brasil vem se preocupando com as políticas públicas para o controle da tuberculose. Em 1975, o Ministério da Saúde do Brasil elaborou o Programa Nacional de Controle da Tuberculose, que tem sofrido reveses ao longo das administrações públicas. A busca de aumento de cobertura, expandindo o atendimento aos pacientes a todos os municípios do País, o diagnostico de no mínimo $92 \%$ dos casos esperados, e o tratamento, com sucesso, de pelo menos $85 \%$ deles, fazem parte das estratégias para o controle da tuberculose ${ }^{3}$. Nos outros $15 \%$ estão incluídos os abandonos, as transferências e os óbitos. O número de casos novos diagnosticados avalia a capacidade de detecção dos serviços de saúde e a tendência da endemia, enquanto a letalidade pode estimar a precocidade com que os casos são diagnosticados e a adequação do tratamento ${ }^{4}$.

O registro dos casos diagnosticados e de sua evolução são essenciais para o acompanhamento das metas traçadas e para a implementação dos ajustes necessários. No Brasil, o Sistema Nacional de Agravos de Notificação $\left(\right.$ SINAN) ${ }^{5}$ e o Sistema de Informação em Mortalidade (SIM) são os sistemas oficiais de informação das doenças de notificação compulsória, inclusive da tuberculose e de óbitos, respectivamente. Em For- 
taleza, o fluxo geral de informações sobre casos de tuberculose e declarações de óbito consiste no envio dos dados gerados nas unidades de saúde (US) às Secretarias Executivas Regionais (SER), onde são processados e remetidos à Secretaria Municipal de Saúde de Fortaleza (SMSF), que consolida todas as informações das seis SER do município.

Esse estudo tem como objetivo verificar se existe subnotificação de casos de tuberculose que evoluíram para o óbito.

\section{Metodologia}

Estudo descritivo de dados secundários de óbitos obtidos do Sistema de Informação em Mortalidade (SIM) no período de 1999 a 2003, e de dados de notificação provenientes do Sistema de Informação de Agravos de Notificação (Sinan) do município de Fortaleza, no período de 1995 a 2003. Foram considerados óbitos associados à tuberculose aqueles que apresentaram os códigos A15 a A19 e suas seqüelas na categoria B90 da Décima Revisão da Classificação Estatística Internacional de Doenças e Problemas relacionados à Saúde (CID 10), em qualquer uma das linhas da declaração de óbito ${ }^{6}$. Os bancos de dados continham informações digitadas até 19/07/2004. Os bancos de dados foram comparados entre setembro e novembro de 2004.

Os arquivos do SIM e Sinan foram importados para planilhas eletrônicas, onde foram feitas as buscas e comparações das variáveis comuns aos dois bancos de dados, tanto pelo método eletrônico quanto manualmente. Buscou-se localizar no Sinan, os casos registrados no SIM, tendo tuberculose como uma das causas múltiplas do óbito, comparando seus nomes, datas de nascimento e nomes das mães. Quando houve coincidência no nome, a data de nascimento, o nome da mãe, o endereço, a data do início do tratamento e a data do óbito também foram conferidos. A mesma conduta foi adotada quando a data do nascimento ou o nome da mãe foram usados como parâmetro principal de comparação.
A população utilizada para o cálculo de mortalidade foi fornecida pelo Instituto Brasileiro de Geografia e Estatística - IBGE, e disponibilizada através da página do Datasus?.

Foram calculados as médias, medianas e desvios padrões dos números de óbitos informados no SIM, casos notificados no Sinan e de suas faixas etárias; e as semelhanças avaliadas pelo qui quadrado o Epi-info 6.04 (CDC Atlanta, USA).

O projeto foi aprovado pelo Comitê de Ética da Faculdade de Medicina da Universidade Federal do Ceará.

\section{Resultados}

Entre 1999 e 2003, foram registrados 72.260 óbitos no Sistema de Informação em Mortalidade (SIM) do município de Fortaleza, sendo 3.779 (5,2\%) por doenças infecciosas classificadas no capítulo I do Código Internacional de Doenças (CID 10) ${ }^{9}$, e 610 $(0,8 \%)$ que tiveram tuberculose como uma das causas múltiplas associadas. Em 438 $(71,8 \%)$ delas, a tuberculose foi causa básica do óbito e 360 (82,2\%) eram residentes em Fortaleza. Em 90 (14,8\%), a causa básica foi síndrome de imunodeficiência adquirida, em 26 (4,5\%) foi neoplasia e em 56 (8,7\%) foram outras causas. Considerando esses casos, a mortalidade por tuberculose por cem mil habitantes foi de 3,9 em 1999, 2,9 em 2000, 3,5 em 2001, 3,9 em 2002 e 2,3 em 2003. A mortalidade média por tuberculose por cem mil habitantes do período foi de 3,3 óbitos por cem mil habitantes. Em relação a $1999 \mathrm{e}$ 2001, houve redução da mortalidade ( $p=0,04)$, sem diferença em relação aos outros anos ( $\mathrm{p}>0,05)$.

Apenas 204 (33,4\%) casos de tuberculose associados ao óbito identificados no SIM foram notificados pelo Sinan, havendo necessidade de acréscimo das outras 406 (66,6\%), o que representará um aumento de 5,9\% nos casos notificados no período. A proporção de notificações manteve-se relativamente constante entre 1999 e 2002, com média de $37,0 \%$, tendo queda para 19,3\% em 2003 ( $\mathrm{p}=0,03)$ (Gráfico 1). 


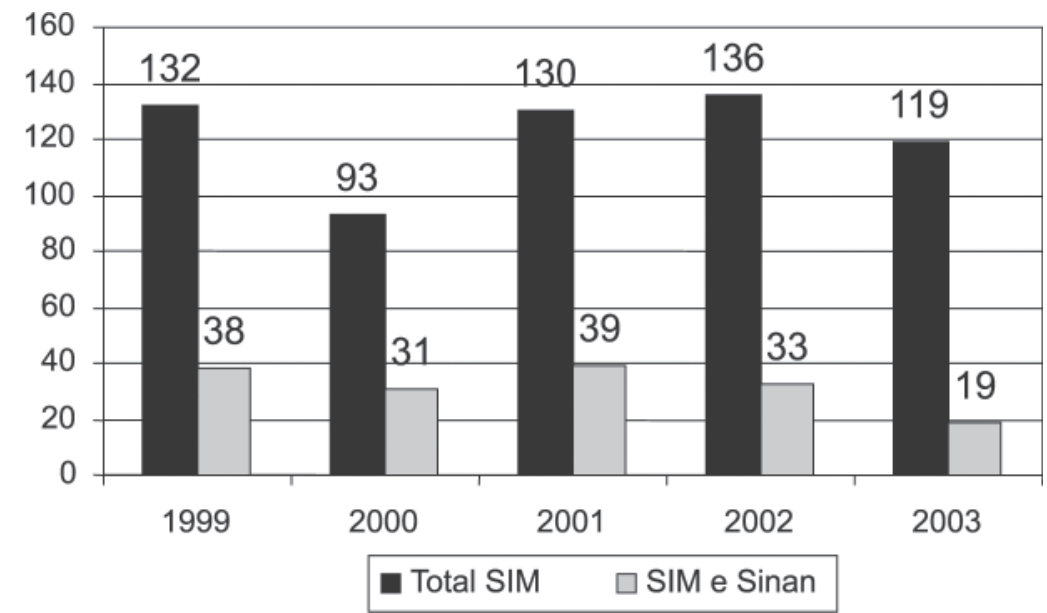

Gráfico 1 - Casos de tuberculose informados no SIM e casos informados no SIM e Sinan simultaneamente, Fortaleza-CE, 1999-2003

Chart 1 - Cases of tuberculosis reported to SIM and cases reported to SIM and Sinan simultaneously, Fortaleza-CE, 1999-2003

Óbitos associados à tuberculose foram registrados em todas as faixas etárias, desde menores de 01 ano até maiores de 90 anos (Tabela 1). A mediana e média de idade foram de 49 anos $(\mathrm{DP} \pm 18,9)$. A moda entre os

Tabela 1 - Óbitos por tuberculose por faixa etária e sexo, Fortaleza-CE, 1999-2003

Table 1 - (Deaths due to tuberculosis by age group and gender, Fortaleza-CE, 1999-2003)

\begin{tabular}{lrrr}
\hline Faixa etária & Feminino & Masculino & Total \\
\hline$<1 a$ & 1 & 1 & 2 \\
$1-4$ & 2 & 4 & 6 \\
$5-9$ & 1 & 2 & 3 \\
$10-14$ & 3 & 3 & 6 \\
$15-19$ & 6 & 5 & 11 \\
$20-24$ & 6 & 13 & 19 \\
$25-29$ & 15 & 28 & 43 \\
$30-34$ & 23 & 35 & 58 \\
$35-39$ & 13 & 51 & 64 \\
$40-44$ & 13 & 36 & 49 \\
$45-49$ & 17 & 35 & 52 \\
$50-54$ & 17 & 43 & 60 \\
$55-59$ & 18 & 29 & 47 \\
$60-64$ & 20 & 30 & 50 \\
$65-69$ & 10 & 25 & 35 \\
$70-74$ & 10 & 28 & 38 \\
$75-79$ & 11 & 17 & 28 \\
80 e mais & 21 & 14 & 35 \\
Ign & 2 & 2 & 4 \\
\hline Total & 209 & 401 & 610 \\
\hline
\end{tabular}

não notificados também foi de 35 a 39 anos, a média foi de 50,2 (DP $\pm 19,6)$ e a mediana de 49 anos. Entre os não notificados, a mediana e a média foram de 47 anos (DP $\pm 17,4)$. A média de idade dos notificados foi maior que a dos não notificados ( $\mathrm{p}<0,049$ ).

Tiveram tuberculose como causa associada ao óbito 209 (34,3\%) pessoas do sexo feminino e 401 (65,7\%) do masculino. Foram notificados 66 (31,6\%) óbitos de pessoas do sexo feminino e $128(34,4 \%)$ do sexo masculino. Ocorreram 497 (81,5\%) óbitos em 42 estabelecimentos de saúde; em 113 $(18,5 \%)$ não havia informação de estabelecimento. Em cinco hospitais ocorreram 324 $(53,1 \%)$, e eles também foram responsáveis por 114 (55,9\%) das notificações.

Entre os óbitos que tiveram tuberculose como causa básica, a localização respiratória sem confirmação bacteriológica foi diagnosticada em 374 (85,4\%) casos, e em 347 (79\%) a localização foi pulmonar. Em apenas $10(2,3 \%)$ casos a tuberculose foi codificada como tendo tido confirmação microbiológica. Doze pessoas faleceram por tuberculose do sistema nervoso central, sendo $4(25,0 \%)$ por meningite. A tuberculose miliar foi responsável por 33 (7,5\%) óbitos e a tuberculose de outros órgãos por 9 (2,1\%). A proporção de casos notificados variou de $16,7 \%$, nas tuberculoses do sistema nervoso 
central, a 33,7\% nas tuberculoses respiratórias não confirmadas. A média de notificações foi de $26,5 \%$ (DP \pm 7 ).

O Sinan registrou 153 óbitos no período de 1999 a 2003 (1999 - 58, 2000 - 50, 2001 $40,2002-23$ e 2003 -Zero). Houve a coincidência de apenas 48 casos que estavam simultaneamente registrados no SIM e no Sinan, como tendo evoluído para o óbito. O acréscimo da evolução para o óbito e do registro dos casos não informados acarretará um aumento de $265,4 \%$ no total de evoluções para o óbito entre os casos registrados no Sinan.

\section{Discussão}

Apenas um terço dos casos que tiveram tuberculose como uma das causas do óbito foi notificado no Sinan. E dos 160 que estavam notificados no Sinan, somente 48 (30\%) tinham o registro da evolução para o óbito. O SIM foi uma importante fonte de resgate de casos graves de tuberculose, visto que as unidades ambulatoriais, que são a principal fonte de informação do Sinan, nem sempre tomam conhecimento dos óbitos dos pacientes com tuberculose, mesmo daqueles que são acompanhados ambulatorialmente. No Rio de Janeiro, em 1998, 41,7\% dos óbitos registrados no SIM haviam sido notificados no Sinan ${ }^{8}$.

A mortalidade e a letalidade da tuberculose são parâmetros importantes para a avaliação da gravidade da endemia, do retardo na detecção de casos, do início do tratamento e da sua efetividade. A falta de notificação dos casos que evoluíram para o óbito pode sugerir que estes pacientes só foram diagnosticados na forma mais avançada, quando foi necessária internação para o diagnóstico e tratamento. O mais sério, quando se considera que quase $80 \%$ dos casos em que a tuberculose foi causa básica do óbito, era o fato da forma ser pulmonar, teoricamente fácil de ser diagnosticada e possivelmente transmissível. Diferente do que aconteceu em São Paulo, em 1998, quando a Aids foi causa básica em $65,3 \%$ dos óbitos ${ }^{9}$, em Fortaleza a Aids foi responsável por $4,5 \%$ das causas básicas associadas à tuberculose.
A subnotificação ocorreu apesar da recomendação de que todo caso confirmado de tuberculose, seja por critério bacteriológico, epidemiológico ou clínico, deve ser notificado, quer o paciente vá continuar o tratamento no local em que está sendo diagnosticado, quer vá ser encaminhado para outro local. Uma outra recomendação tem sido que, ao se codificar um óbito por doença de notificação compulsória, deve-se conferir se ele está notificado no Sinan; se não estiver, deve ser notificado. Possivelmente, essas informações precisam ser reforçadas junto aos profissionais que compõem os núcleos de vigilância epidemiológica hospitalares, comissões de controle de infecção hospitalar e codificadores de declarações de óbito. É possível que uma forma de "comunicação” mais eficiente entre o SIM e o Sinan possa minorar a subnotificação, talvez através da conferência automática dos dados da declaração de óbito registrados no SIM com aqueles do Sinan, e informação dos casos não registrados para posterior investigação e digitação.

Entre as limitações deste estudo citamse os erros de digitação, como o uso de dois espaços entre os sobrenomes e abreviaturas de nomes, o que pode tornar maior a subnotificação. As diferenças nas seqüências com que as datas são apresentadas no SIM (dia/mês/ano) e no Sinan (ano/mês/ dia), como acontece com a data de nascimento, representaram outra dificuldade. Os bancos de dados de óbitos e de casos continham informações digitadas até 19/07/ 2004. É possível que haja declarações de óbito de 2003, e mesmo de fichas de investigação de casos de tuberculose que ainda não foram registrados, podendo ainda haver acréscimos nesses bancos de dados, o que pode falsear conclusões sobre a redução de casos e de mortalidade. Não foi possível detectar situações específicas que determinassem a subnotificação, visto que os hospitais notificaram parte dos casos e deixaram de informar outros, também não houve diferença entre os sexos e mesmo as diferenças entre as médias de idade foram discretas. 
Considerando o aumento na taxa de mortalidade por tuberculose em diversas áreas do globo ${ }^{10}$, é importante que se esteja atento às declarações de óbito como fonte de informação de casos de tuberculose e de sua evolução.

\section{Conclusão}

Houve grande subnotificação dos casos de tuberculose que evoluíram para o óbito, o que pode contribuir para uma falsa sensação de redução de casos e de sua gravidade quando se analisa a tuberculose através do Sinan.

\section{Agradecimentos}

ÀEquipe de Vigilância Epidemiológica da Secretaria Municipal de Saúde de Fortaleza pela disponibilização dos dados.

\section{Referências}

1. Ruffino-Netto A. Tuberculose: a calamidade negligenciada. Rev Soc Bras Med Trop 2002 ; 35(1): 518 .

2. Brasil, Ministério da Saúde. Secretaria Executiva: Datasus. Informações de saúde: mortalidade [on-line]. Disponível em http://www.datasus.gov.br

3. Brasil. Ministério da Saúde. Controle da Tuberculose: uma proposta de integração ensino-serviço. Funasa, $5^{\mathrm{a}}$ ed. Rio de Janeiro; 2002.

4. Brasil. Ministério da Saúde. Tuberculose: Guia de Vigilância Epidemiológica. Funasa. Brasília; 2002, $100 \mathrm{p}$.

5. Brasil. Ministério da Saúde. Capacitação no uso do Sistema Nacional de Agravos de Notificação - SINAN Tuberculose. Brasília; 2003.

6. Centro Brasileiro de Classificação de Doenças. CID 10 Classificação Estatística Internacional de Doenças e Problemas Relacionados à Saúde - 10 a Revisão. Editora da Universidade de São Paulo; 1999.
7. Brasil, Ministério da Saúde. Secretaria Executiva: Datasus. Informações de saúde: informações demográficas e socioeconômicas: População residente [on-line]. Disponível em http://www.datasus.gov.br

8. Selig L, Belo M, Cunha AJLA, Teixeira EG, Brito R, Luna AL, Trajman A. Óbitos atribuídos à tuberculose no Estado do Rio de Janeiro. J Bras Pneumol 2004; 30(4): 417-24.

9. Santo AH, Pinheiro CE, Jordani MS. Causas múltiplas de morte relacionadas à tuberculose no estado de Sao Paulo, 1998. Rev Saúde Pública 2003; 37(6): 714-21.

10. Antunes JLF, Waldman EA. Tuberculosis in the twentieth century: time-series mortality in São Paulo, Brazil, 1900-97. Cad Saúde Pública 1999, 15(3): 463-76.

recebido em: 08/09/04 versão final reapresentada em: 20/12/04 aprovado em: 25/01/05 